Erschienen in: Wimmer, Rainer (Hrsg.): Das 19. Jahrhundert: Sprachgeschichtliche Wurzeln des heutigen Deutsch. - Berlin, New York: de Gruyter, 1991. S. 8-11. (Institut für deutsche Sprache.

\title{
Jahrbuch 1990)
}

\section{Eröffnungsansprache des Präsidenten des Instituts für deutsche Sprache}

Meine sehr verehrten Damen und Herren,

Zur Jahrestagung 1990 des Instituts für deutsche Sprache heiße ich Sie, die in groBer Zahl aus 24 Ländern gekommen sind, herzlich willkommen. Mein besonderer Gruß gilt den Vertretern der Stadt Mannheim, Herrn Oberbürgermeister Gerhard Widder und Herrn Bürgermeister Mark, Herrn Prorektor Groth von der Universität Mannheim, dem Direktor des Zentralinstituts für Sprachwissenschaften an der Ostberliner Akademie der Wissenschaften, Herrn Viehweger, dem Direktor des Goethe-Instituts Mannheim, Herrn Kage und dem Herrn Vorsitzenden der Freundengesellschaft des Instituts, Herrn Konrad Beyer.

Den Grüßen füge ich den Dank des Kuratoriums für die Vorbereitung der Tagung an. Er gilt den Herren Cherubim, von Polenz, Schwitalla und Wimmer als Mitglieder des Vorbereitungsausschusses, der für das Vortragsprogramm verantwortlich zeichnet, und den Damen und Herren des Instituts, die für die Organisation und den technischen Ablauf sorgen.

Pünktlich zum Beginn dieser Tagung ist das Jahrbuch 1989 erschienen, das auf dem Umschlag den Titel der letztjährigen Vortragssammlung 'Deutsche Gegenwartssprache - Tendenzen und Perspektiven' mit den folgenden Worten erläutert: „Mit der Erforschung und Dokumentation des heutigen Deutsch befaßt sich das Institut für deutsche Sprache seit seiner Gründung im Jahr 1964. Diesem wissenschaftlichen Dauerauftrag entsprechend war die internationale Tagung zum 25-jährigen Jubiläum des Instituts der deutschen Gegenwartssprache und ihrer Entwicklung gewidmet." Werden wir also im ersten Jahr nach dem Jubiläum wortbrüchig, wenn wir uns jetzt dem 19. Jahrhundert zuwenden und die Tagung einem historischen Generalthema widmen, unter dem Vorträge und Diskussionen stattfinden werden, die nach Wurzeln des heutigen Deutsch in dieser Zeit fragen? Wir erweitern das erste Mal den Untersuchungsraum unserer Sprachbetrachtung, und das ist nötig.

Je weiter wir uns zeitlich vom Ende des Zweiten Weltkrieges entfernen, um so problematischer wird die Periodisierungszäsur für den Begriff Gegenwartssprache, deren Beginn wir noch immer bei 1945 ansetzen und die jetzt bereits einen Zeitraum von 45 Jahren, also von mehr als einer Generation umfaßt. Unserer Arbeit auf dem Gebiete der synchronischen Sprachanalyse ist unmerklich die diachronische Dimension hinzugewachsen. Diese Gedanken über die Verwerfungen der zeitlichen Perspektive lassen auch die zwölf Jahre zwischen 1933 und 1945 sehr kurz erscheinen, 
so daß man sich die Größe und Tragweite dieses weltpolitischen Verhängnisses keinesfalls nur so kurzfristig entstanden erklären kann. Man muß die Herrschaftsjahre Hitlers überschreiten, um diesen Block der 12 nationalsozialistischen Jahre gedanklich, ideologisch, faktisch und sprachlich in die Entwicklung einpassen zu können, und das heißt für uns, wir haben in der Sprachgeschichte die Vorbereitungen vor 1933 schonungslos aufzudecken wie die Nachwirkungen seit 1945 . Hierbei gelangt man jenseits des Jahres 1933 unweigerlich über die Jahrhundertgrenze hinweg in die Zeit unserer Groß- und Urgroßeltern.

Das 19. Jahrhundert ist eine ereignisreiche Zeit, die von einem aternberaubenden, oft heterogenen Wechsel der Ideen, Empfindungen und Ereignisse geprägt worden ist und nicht von der idyllischen Beschaulichkeit der Stahlstiche Ludwig Richters oder der Spitzwegschen Bilder.

Die Napoleonischen Eroberungsfeldzüge haben Europa entscheidend verändert. Die Völkerschlacht bei Leipzig, 1813, die Revolution 1848, die Kriege 1864, 1860 und 1870 zeigen einen unruhigeren politischen und militärischen Pulsschlag der Zeit, als man im Gedächtnis haben mag. Die Reichsgründung, der Imperialismus und die Koloniale Expansion sind politische Aktivitäten, die weit über Europa hinausgreifen. Die aus der Kohleförderung neuerschlossenen Energiequellen Dampf, Gas und Elektizität setzen mit zunehmender Beschleunigung die Industrialisierung in Gang und führen zu komplexen sozialen Bewegungen der Landflucht, der Verstädterung und der Massenkultur. Der Bau von Straßen, Schienen und Kanälen knüpft das Verkehrsnetz für den Handel dichter und setzt Produktion und den Verksuf in schnellere Rotation; dazu treten als Katalysatoren des Nachrichtenaustausches die Verbesserung des Postwesens mit Brief, Telegramm, Postkarte und Ferngesprächen. Die Bevölkerung gewinnt eine ihr bisher unbekannte körperliche und geistige Mobilität. Denn das Schulwesen wird im Laufe des Jahrhunderts auf- und ausgebaut und in erstaunlicher Weise, vor allem für die berufliche Bildung, differenziert. Erstmals in der deutschen Sprachgeschichte kann am Ende des Jahrhunderts fast die gesamte Bevölkerung lesen und schreiben, natürlich in unterschiedlichen Graden, aber doch so, daß jetzt ungeahnte Bildungsmöglichkeiten bestehen. Es wachsen das Leseangebot ebenso wie das Lesebedürfnis und die Leseerfahrung, wie auch das Verlangen und die Notwendigkeit der schriftlichen Mitteilung. Ein dichtes Zeitungs- und Zeitschriftennetz wird immer weiter ausgebaut und dank neuer Erfindungen schneller und aktueller. Die Fotografie beginnt als Nachrichtenträger ihre textbegleitende semiotische Aufgabe wahrzunehmen. Neben den Schulen aller Typen werden Universitäten, Technische Hochschulen, Fachhochschulen, Theater, Museen und Dichtungsvereine 
gegründet. Man versucht, der Expansion und Explosion des Wissens Herr zu werden und die großen Mengen an Erkenntnissen verfügbar zu machen: z.B. durch die Gründung von Konversationslexika, Enzyklopädien und großen Wörterbüchern. Es werden umfangreiche wissenschaftliche und Volksbibliotheken gegründet, Leihbüchereien wachsen wie Pilze aus dem Boden und verbreiten triviale Literatur, die jetzt die Lektüre großer Bevölkerungsschichten erweitert und diese vom intensiven Lesen immer der gleichen wenigen Bücher zum extensiven Lesen immer neuer Inhalte führt. In der Literatur verzeichnen wir die Epochen der Klassik, Romantik, des Vormärz, des poetischen Realismus, des Naturalismus, Impressionismus und Expressionismus. Die Anregungen bedeutsamer Philosophen wie Kant, Schelling, Hegel, Marx und Nietzsche wirken weit über die Jahrhundertgrenze bis in unsere Gegenwart hinein.

Die Differenzierung der technischen und naturwissenschaftlichen Disziplinen und der Handwerksberufe bringen die weite Auffächerung der Fachsprachen darunter auch der Wissenschaftssprachen mit sich. Unsere Sprache entfaltet die ihr eigenen Möglichkeiten der elastischen Wortbildung, um die Erweiterung des Lexikons zu fassen.

Parallel zu den genannten Ereignissen ist das deutsche Nationalbewußtsein gewachsen und systematisch in der schulischen und militärischen Erziehung der Jugend gelehrt und internalisiert worden. Die politischen Erfolge werden mit entsprechend interpretierten Beispielen der deutschen Geschichte, besonders aus der Kaiserzeit des Mittelalters, gerechtfertigt und mit darwinistischer Konsequenz als zwangsläufige Entwicklung auf die vermeintlichen historischen Quellen zurückgeführt. So ist ein mit überheblichen Qualitätsansprüchen angreichertes Deutschlandbild, begleitet von ersten Strömungen des Antisemitismus, entstanden, das eine der verhängnisvollen Voraussetzungen für die beiden Weltkriege und für die Gründung des Dritten Reichs geworden ist und dessen Ideen gerade heute bei unseren europäischen Nachbarn lebendiger und bedrohlicher in Erinnerung sein dürften als bei vielen von uns.

Es ist zu Beginn einer wissenschaftlichen Tagung eigentlich nicht zu verantworten, mit schnellen Strichen oberflächlich das Bild eines ganzen Jahrhunderts zu skizzieren. Aber ich wollte mit diesem Stenogramm ein Assoziationsfeld wachrufen, um zu zeigen, wie viele und wie bedeutsame Verbindungen aus dem 19. Jahrhundert in das 20. herüberführen und weiterwirken und wie viele ungelöste Probleme als beschwerende Hypothek liegengeblieben sind und uns belasten.

Mit dieser Jahrestagung beginnen wir, uns für die jüngste Sprachgeschichte zu öffnen. Die Jahrestagung 1992 wird unter dem Thema 
"Deutsch als Verkehrs- und Institutionen-Sprache in Europa" stehen. Sie wird die Gelegenheit geben, die Situation der deutschen Sprache aus dem Blickwinkel aller deutschsprachigen Länder zu beleuchten. DaB der gegenseitigen Zusammenarbeit dank der jüngsten Entwicklung keine hindernden Grenzen mehr entgegenstehen, ist für unser Fach ein überraschendes und besonders glückliches Ereignis.

Auf dem Plakat zur Tagung ist eine Maschine abgebildet, bei der man nicht recht sieht, ob es sich um eine Lokomotive oder um eine Lokomobile handelt. Für unsere geistige Beweglichkeit sollten beide Symbole wichtig sein: Mit dem Wunsch, die Gedanken und Diskussionsbeiträge orts- und fernbeweglich zu halten, eröffne ich die Tagung.

Siegfried Grosse 